\title{
A Note on Laws of Motion for Aggregate Distributions
}

\author{
Damir Stijepic \\ Johannes Gutenberg University, Mainz, Germany \\ Email: mail@damir.stijepic.com
}

How to cite this paper: Stijepic, D. (2020) A Note on Laws of Motion for Aggregate Distributions. Theoretical Economics Letters, $10,1358-1371$.

https://doi.org/10.4236/tel.2020.106083

Received: October 18, 2020

Accepted: December 22, 2020

Published: December 25, 2020

Copyright (C) 2020 by author(s) and Scientific Research Publishing Inc. This work is licensed under the Creative Commons Attribution International License (CC BY 4.0).

http://creativecommons.org/licenses/by/4.0/

\begin{abstract}
I derive the law of motion for the aggregate distribution directly from the laws of motion for the individuals' states. By relying on concepts from measure theory, the derivation is concise and intuitive. I address random shocks both at the micro level and at the macro level. Micro-level shocks completely cancel at the aggregate level provided that a law of large numbers applies. Therefore, the law of motion for the aggregate distribution is a deterministic process in the absence of macro-level uncertainty. If there are macro-level risks, the law of motion for the aggregate distribution exhibits a stochastic component additionally. I illustrate the formalism in a model of wealth accumulation with stochastic interest rates, deriving the law of motion for the aggregate wealth distribution.
\end{abstract}

\section{Keywords}

Aggregate Distribution, Law of Motion, Kolmogorov Forward Equation, Fokker-Planck Equation, Wealth Distribution

\section{Introduction}

Nowadays, many macroeconomic models depart from the assumption of a representative agent and acknowledge the importance of heterogeneity among individuals. A central question is how the behavior of various heterogeneous agents translates into aggregate outcomes. How are individual consumption and savings decisions related to the distribution of wealth? How do individual earnings profiles determine the distribution of wages?

The aggregate distribution indicates the shares of the population that are in specific states. These shares are known in the initial period. However, from the perspective of the initial period, the exact future trajectory of the aggregate distribution may be known or unknown-depending on the structure of the 
economy and, in particular, the nature of the individuals' stochastic processes. It is useful to distinguish risks at the macro level and at the micro level. Macro-level risks are the common random shocks that affect a significant portion of the population. Micro-level risks occur at the individual level, affecting only a negligible portion of the population. Furthermore, the micro-level risks of the individuals in the economy are conditionally independent random variables given the macro-level shocks. An important effect of aggregation is that the micro-level risks completely cancel at the aggregate level provided that a law of large numbers applies. In the absence of macro-level uncertainty, the exact future trajectory of the aggregate distribution is known and the associated law of motion for the aggregate distribution is a deterministic process. However, if there are macro-level risks, one may only obtain a deterministic aggregate distribution conditional on the macro-level shocks. In that case, the exact future trajectory of the aggregate distribution is unknown and the associated law of motion for the aggregate distribution is a stochastic process.

In the present paper, I derive the law of motion for the aggregate distribution directly from the individuals' laws of motion, addressing random shocks both at the micro level and at the macro level. Specifically, if the shocks at the individual level are independently and identically distributed and if there are sufficiently many individuals in the economy, the law of motion for a person's probability function coincides with that for the aggregate distribution. While the former distribution represents probabilities of specific events, the latter distribution represents shares of the population in specific states. If there is also macro-level uncertainty, the law of motion for a person's probability function does not coincide with that for the aggregate distribution in general. It is the probability function conditional on the macro-level shocks that yields the law of motion for the aggregate distribution. The law of motion for the aggregate distribution exhibits a stochastic component in the latter case.

My paper is most closely related to the literature in economics that makes use of the Fokker-Planck equations or the Kolmogorov forward equations in order to describe the distributions generated by stochastic processes (e.g., Achdou et al., forthcoming; Bayer \& Wälde, 2010; Moscarini, 2005; Merton, 1975). I complement the literature by providing a concise and intuitive derivation that relies on concepts from measure theory.

This paper is structured as follows. In Section 2, I present a concise and intuitive formalism for deriving laws of motion for aggregate distributions. In Section 3, I illustrate the formalism in a model of wealth accumulation with stochastic interest rates, deriving the law of motion for the aggregate wealth distribution. Section 4 draws some conclusions. The proof of Corollary 1 is in Appendix A. An illustrative example with a discrete state space is in Appendix B.

\section{Theoretical Foundation}

Let the state of a person $i$, denoted by $s^{i}$, be summarized by the $n$-dimensional 
real-valued vector $\left(s_{1}^{i}, s_{2}^{i}, \cdots, s_{n}^{i}\right) \in \Omega \subset \mathbb{R}^{n}$. The Borel $\sigma$-algebra $\mathcal{B}$ on the state space $\Omega$ contains the relevant events $B \subset \Omega$. The probability function $P^{i}(\cdot)$ is a Borel measure that assigns probabilities to all the events $B \in \mathcal{B}$. I assume that the probability distribution admits a differentiable probability density function $p^{i}(\cdot)$ that assigns densities to the states $s^{i} \in \Omega$.

As it is standard in continuous-time analysis, I introduce the concept of the infinitesimal time period $d t>0$. The eventual modeling equations will be those that emerge in the limit as $d t$ approaches zero. Let the evolution over time of the person's state be described by the Markovian stochastic process $s_{d t}^{i}\left(s^{i}\right)$. In particular, a person's current state $s^{i}$ determines the probability that the person's state $s_{d t}^{i}$ will be in the set $B$ at the future point in time $d t$, denoted by $P_{d t}^{i}(B)$.

The properties of the stochastic process for the person's state $s_{d t}^{i}(\cdot)$ are typically expressed in the form of a stochastic differential equation, i.e., a law of motion. An expression for the probability function $p_{d t}^{i}(\cdot)$ that is consistent with the stochastic process $s_{d t}^{i}(\cdot)$ is typically not readily available. The remainder of this section is structured as follows. In Section 2.1, I characterize the probability function $p_{d t}^{i}(\cdot)$ that is consistent with the stochastic process for the person's state $s_{d t}^{i}(\cdot)$. I derive the law of motion for the probability function $p^{i}(\cdot)$ in Section 2.2. I discuss the aggregation of the individuals' probability functions in Section 2.3 .

\subsection{Probabilities and the Probability Function}

In this section, I characterize the probability function $p_{d t}^{i}(\cdot)$ that is consistent with the stochastic process $s_{d t}^{i}(\cdot)$ and the respective stochastic differential equation. By construction, the probability that a person's state will be in the set $B$ at the future point in time $d t, P_{d t}^{i}(B)$, equals the integral of the density function $p_{d t}^{i}(\cdot)$ over the set $B$, i.e.,

$$
P_{d t}^{i}(B)=\int_{B} p_{d t}^{i}\left(s^{i}\right) d s^{i}=\int_{\Omega} p_{d t}^{i}\left(s^{i}\right) \mathbb{1}_{B}\left(s^{i}\right) d s^{i},
$$

where $\mathbb{1}_{B}\left(s^{i}\right)$ is an indicator function that equals one if the state $s^{i}$ belongs to the set $B$, i.e., $s^{i} \in B$, but is zero otherwise. An alternative expression for the probability that a person's state will be in the set $B$ at the future point in time $d t$ is

$$
P_{d t}^{i}(B)=\int_{\Omega} p^{i}\left(s^{i}\right) \mathbb{E}_{B}\left(s_{d t}^{i}\left(s^{i}\right)\right) d s^{i},
$$

where $\mathbb{E}$ denotes expectations. Intuitively, the probability conditional on the current state $s^{i}$ that the person's state $s_{d t}^{i}$ will be in the set $B$ is simply $\mathbb{E} \mathbb{1}_{B}\left(s_{d t}^{i}\left(s^{i}\right)\right)$. Furthermore, the unconditional probability that a person's state $s_{d t}^{i}$ will be in the set $B$ equals the integral of the conditional probabilities weighted by the initial probability function.

An important feature of Equation (2) in contrast to Equation (1) is that expressions for the terms on the right-hand side are readily available. Equating 
Equation (1) and Equation (2), I obtain a recursive formula for the density function $p_{d t}^{i}(\cdot)$

$$
\int_{\Omega} p_{d t}^{i}\left(s^{i}\right) \mathbb{1}_{B}\left(s^{i}\right) d s^{i}=\int_{\Omega} p^{i}\left(s^{i}\right) \mathbb{E} \mathbb{1}_{B}\left(s_{d t}^{i}\left(s^{i}\right)\right) d s^{i} .
$$

A key question is whether the condition in Equation (3) is sufficient to characterize the probability function $p_{d t}^{i}(\cdot)$ that is consistent with the stochastic process $s_{d t}^{i}(\cdot)$. The following corollary from measure theory provides guidance.

Corollary 1 The two Borel measures $P(d x)$ and $\tilde{P}(d x)$ are equivalent if and only if

$$
\int \mathbb{1} P(d x)=\int \mathbb{1} \tilde{P}(d x) \quad \forall \mathbb{1} \in \mathcal{H}
$$

where $\mathcal{H}$ is the set of open set indicator functions.

By Corollary 1, knowing a Borel measure on the open sets is sufficient to fully characterize the measure. Hence, the probability function $p_{d t}^{i}(\cdot)$ is consistent with the stochastic process for the person's state $s_{d t}^{i}(\cdot)$ if the probability implied by the probability function $p_{d t}^{i}(\cdot)$ in Equation (1) coincides with that implied by the stochastic processes $s_{d t}^{i}(\cdot)$ in Equation (2) for any open set $B$, i.e., if the condition in Equation (3) is satisfied for any open set $B$.

\subsection{The Evolution over Time of the Probability Function}

In this section, I derive the law of motion for the probability function $p^{i}(\cdot)$. The starting point is the condition in Equation (3), which has to hold for any arbitrary open set $B$. Let the indicator function $\mathbb{1}_{B}(\cdot)$ on the right-hand side of Equation (3) be separable. Specifically, the function $F(\cdot)$ exists so that the right-hand side of Equation (3) is equivalent to $\int_{\Omega} F\left(s^{i}, d t\right) \mathbb{1}_{B}\left(s^{i}\right) d s^{i} \cdot{ }^{1}$ After collecting terms, Equation (3) simplifies to

$$
\int_{\Omega}\left\{p_{d t}^{i}\left(s^{i}\right)-F\left(s^{i}, d t\right)\right\} \mathbb{1}_{B}\left(s^{i}\right) d s^{i}=0
$$

The condition in Equation (4) is obviously satisfied for any arbitrary open set $B$ if the expression in brackets vanishes. In other words, a probability function $p_{d t}^{i}(\cdot)$ is consistent with the stochastic process for the person's state $s_{d t}^{i}(\cdot)$ if $p_{d t}^{i}\left(s^{i}\right)-F\left(s^{i}, d t\right)=0$ for (almost) all $s^{i} \in \Omega$.

As the time period $d t$ approaches zero, one obtains the law of motion for the probability function $p^{i}(\cdot)$. This law of motion is the so-called Fokker-Planck equation or Kolmogorov forward equation for the stochastic process $s_{d t}^{i}(\cdot)$. The law of motion for the probability function $p^{i}(\cdot)$ is typically a partial differential equation in time and in the person's state.

\subsection{Aggregation}

From the perspective of the initial period, the exact future trajectory of a person's stochastic process $s_{d t}^{i}(\cdot)$ is unknown in general. While the realization of the state in the initial period is known, only probabilistic statements about the

${ }^{1}$ Typically, it suffices to apply change of variables formulae in order to obtain the function $F(\cdot)$. 
person's future state are possible. In that sense, there is a distribution over the state space already at the individual level, i.e., the probability distribution for the person's future state $p_{d t}^{i}(\cdot)$. In an economy with many individuals, the heterogeneity among the individuals also implies a distribution over the state space, denoted by $p(\cdot)$. This aggregate distribution indicates the shares of the population that are in specific states. These shares are known in the initial period and are, hence, not probabilistic constructs. From the perspective of the initial period, the exact future trajectory of the aggregate distribution may be known or unknown-depending on the structure of the economy and, in particular, the nature of the individuals' stochastic processes $s_{d t}^{i}(\cdot)$.

It is useful to distinguish risks at the macro level and at the micro level. Macro-level risks are the common random shocks that affect a significant portion of the population. Micro-level risks occur at the individual level, affecting only a negligible portion of the population. Furthermore, the micro-level risks of the individuals in the economy are conditionally independent random variables given the macro-level shocks. An important effect of aggregation is that the micro-level risks completely cancel at the aggregate level provided that a law of large numbers applies-in particular, if the shocks at the individual level are essentially pairwise independent and if there are sufficiently many individuals in the economy. In the absence of macro-level uncertainty, a deterministic (almost surely) aggregate distribution arises (see Sun, 2006; Duffie \& Sun, 2012; He et al., 2017). In that case, the exact future trajectory of the aggregate distribution is known and the associated law of motion for the aggregate distribution is a deterministic process. However, if there are macro-level risks, one may only obtain a deterministic (almost surely) aggregate distribution conditional on the macro-level shocks (see Qiao et al., 2016). In that case, the exact future trajectory of the aggregate distribution is unknown and the associated law of motion for the aggregate distribution is a stochastic process.

All in all, if the shocks at the individual level are independently and identically distributed and if there are sufficiently many individuals in the economy, the law of motion for a person's probability function coincides with that for the aggregate distribution. While the former distribution represents probabilities of specific events, the latter distribution represents shares of the population in specific states. If there is also macro-level uncertainty, the law of motion for a person's probability function does not coincide with that for the aggregate distribution in general. It is the probability function conditional on the macro-level shocks that yield the law of motion for the aggregate distribution. Notably, the law of motion for the aggregate distribution exhibits a stochastic component in the latter case.

\section{Wealth Accumulation in a Model with Stochastic Interest Rates}

All individuals in the economy maximize their expected lifetime utility. Specifi- 
cally, the expected lifetime utility of a person $i$ at the point in time $t \in \mathbb{R}^{+}$is

$$
U_{t}^{i}=\mathbb{E} \int_{t}^{\infty} \mathrm{e}^{-\rho(\tau-t)} \ln \left(c_{\tau}^{i}\right) d \tau
$$

where $\rho \in \mathbb{R}^{+}$is the time-preference rate, $c_{\tau}^{i}$ is the consumption of the person $i$ at the point in time $\tau$, and $\mathbb{E}$ denotes expectations. The wealth of a person $i$, denoted by $a^{i}$, evolves according to the stochastic differential equation $d a^{i}=r^{i}\left(a^{i}-c^{i}\right) d t$, where $r^{i}$ denotes the stochastic interest rate that the person $i$ faces. The consumption path is to be chosen optimally subject to the lifetime budget constraint. In particular, $a^{i} \geq 0$ at all times.

A person's interest rate is either low, $r^{i}=\underline{r}$, or high, $r^{i}=\bar{r}$, where $\underline{r}, \bar{r} \in \mathbb{R}^{+}$and $\bar{r}>\underline{r}$. The interest rate jumps to the high value $\bar{r}$ in response to a micro-level shock, modeled by the Poisson process $q_{\lambda}^{i}$ with an arrival rate of $\lambda>0$, or in response to a macro-level shock, modeled by the Poisson process $q_{\mu}$ with an arrival rate of $\mu>0$. I assume that the micro-level shocks are essentially pairwise independent. Formally, a person's interest rate evolves according to the stochastic differential equation

$$
d r^{i}=\left(\bar{r}-r^{i}\right) d q_{\lambda}^{i}+\left(\bar{r}-r^{i}\right) d q_{\mu} .
$$

I note that the high interest-rate state, $\bar{r}$, is an absorbing state. After entering that state, a person will always face the high interest rate with certainty.

The solution to the individual's optimization problem is described by

$$
d a^{i}=\left(r^{i}-\rho\right) a^{i} d t,
$$

i.e., the optimal law of motion for personal wealth that incorporates the optimal consumption and savings decisions. ${ }^{2}$

\subsection{The Probability Function for Personal Wealth and the Interest Rate}

By Corollary 1, a joint probability function for personal wealth and the interest rate, denoted by $p_{d t}^{i}\left(a^{i}, r^{i}\right)$, is consistent with the individual's laws of motion for personal wealth in Equation (6) and for the interest rate in Equation (5) if it satisfies the condition in Equation (3) for all open set indicator functions. Specifically, the condition is

$$
\sum_{r^{i}} \int p_{d t}^{i}\left(a^{i}, r^{i}\right) \mathbb{1}_{B}\left(a^{i}, r^{i}\right) d a^{i}=\sum_{r^{i}} \int p^{i}\left(a^{i}, r^{i}\right) \mathbb{E} \mathbb{1}_{B}\left(s_{d t}^{i}\left(a^{i}, r^{i}\right)\right) d a^{i} .
$$

By the law of motion in Equation (5), the interest rate is expected to jump to the high value $\bar{r}$ and to stay at the initial level $r^{i}$ with a probability of $(\lambda+\mu) d t$ and $1-(\lambda+\mu) d t$, respectively. Hence,

$\mathbb{E} \mathbb{1}_{B}\left(s_{d t}^{i}\left(a^{i}, r^{i}\right)\right)=(\lambda+\mu) d t \mathbb{1}_{B}\left(a^{i}+d a^{i}, \bar{r}\right)+(1-(\lambda+\mu) d t) \mathbb{1}_{B}\left(a^{i}+d a^{i}, r^{i}\right)$, where $d a^{i}=\left(r^{i}-\rho\right) a^{i} d t$.

After applying a change of variables formula in order to integrate over $a^{i}+d a^{i}$ instead of $a^{i}$, the integral on the right-hand side of Equation (7) be-

${ }^{2}$ Recent studies of the impact of interest-rate heterogeneity among the population on wealth inequality include Gabaix et al. (2016), Cao and Luo (2017), and Benhabib and Bisin (2018). 
comes $\begin{array}{ll}\int\left(1+\left(r^{i}-\rho\right) d t\right)^{-1} p^{i}\left(a^{i} /\left(1+\left(r^{i}-\rho\right) d t\right), r^{i}\right) \\ \times\left\{(\lambda+\mu) d t \mathbb{1}_{B}\left(a^{i}, \bar{r}\right)+(1-(\lambda+\mu) d t) \mathbb{1}_{B}\left(a^{i}, r^{i}\right)\right\} d a^{i}\end{array}$ terms so that Equation (7) simplifies to

$$
\sum_{r^{i}} \int \mathbb{1}_{B}\left(a^{i}, r^{i}\right)\left\{p_{d t}^{i}\left(a^{i}, r^{i}\right)-F\left(a^{i}, r^{i}, d t\right)\right\} d a^{i}=0,
$$

where $F\left(a^{i}, \underline{r}, d t\right)=(1-(\lambda+\mu) d t)(1+(\underline{r}-\rho) d t)^{-1} p^{i}\left(a^{i} /(1+(\underline{r}-\rho) d t), \underline{r}\right)$

$$
\text { and } \begin{aligned}
F\left(a^{i}, \bar{r}, d t\right)= & (\lambda+\mu) d t(1+(\underline{r}-\rho) d t)^{-1} p^{i}\left(a^{i} /(1+(\underline{r}-\rho) d t), \underline{r}\right) \\
& +(1+(\bar{r}-\rho) d t)^{-1} p^{i}\left(a^{i} /(1+(\bar{r}-\rho) d t), \bar{r}\right)
\end{aligned}
$$

condition is obviously satisfied for all open set indicator functions if the terms in brackets vanish.

In order to obtain the law of motion for the probability function $p^{i}\left(a^{i}, r^{i}\right)$, I calculate the limit of $\left(p_{d t}^{i}\left(a^{i}, r^{i}\right)-F\left(a^{i}, r^{i}, d t\right)\right) / d t=0$ as the time period $d t$ approaches zero. Specifically, $\lim _{d t \rightarrow 0}\left(p_{d t}^{i}\left(a^{i}, \underline{r}\right)-F\left(a^{i}, \underline{r}, d t\right)\right) / d t=0 \quad$ and $\lim _{d t \rightarrow 0}\left(p_{d t}^{i}\left(a^{i}, \bar{r}\right)-F\left(a^{i}, \bar{r}, d t\right)\right) / d t=0$ yield the laws of motion for the probability function $p^{i}\left(a^{i}, \underline{r}\right)$ and for the probability function $p^{i}\left(a^{i}, \bar{r}\right)$ :

$$
\begin{aligned}
d p^{i}\left(a^{i}, \underline{r}\right)= & -(\underline{r}-\rho) a^{i} \frac{\partial p^{i}}{\partial a^{i}}\left(a^{i}, \underline{r}\right) d t-(\underline{r}-\rho) p^{i}\left(a^{i}, \underline{r}\right) d t \\
& -(\lambda+\mu) p^{i}\left(a^{i}, \underline{r}\right) d t \\
d p^{i}\left(a^{i}, \bar{r}\right)= & -(\bar{r}-\rho) a^{i} \frac{\partial p^{i}}{\partial a^{i}}\left(a^{i}, \bar{r}\right) d t-(\bar{r}-\rho) p^{i}\left(a^{i}, \bar{r}\right) d t \\
& +(\lambda+\mu) p^{i}\left(a^{i}, \underline{r}\right) d t
\end{aligned}
$$

$\partial p^{i} / \partial a^{i}$ denotes the partial derivate of the probability function with respect to personal wealth, i.e.,

$$
\partial p^{i} / \partial a^{i}\left(a^{i}, r^{i}\right)=\lim _{d a^{i} \rightarrow 0}\left(p^{i}\left(a^{i}+d a^{i}, r^{i}\right)-p^{i}\left(a^{i}, r^{i}\right)\right) / d a^{i} .^{3}
$$

${ }^{3} \mathrm{I}$ note that rearranging $\left(p_{d t}^{i}\left(a^{i}, \underline{r}\right)-F\left(a^{i}, \underline{r}, d t\right)\right) / d t=0$ and $\left(p_{d t}^{i}\left(a^{i}, \bar{r}\right)-F\left(a^{i}, \bar{r}, d t\right)\right) / d t=0$ yields:

$$
\begin{aligned}
\frac{p_{d t}^{i}\left(a^{i}, \underline{r}\right)-p^{i}\left(a^{i}, \underline{r}\right)}{d t}= & \frac{p^{i}\left(a^{i}-\frac{(\underline{r}-\rho) a^{i} d t}{1+(\underline{r}-\rho) d t}, \underline{r}\right)-p^{i}\left(a^{i}, \underline{r}\right)}{(1+(\underline{r}-\rho) d t) d t}-\frac{(\underline{r}-\rho) p^{i}\left(a^{i}, \underline{r}\right)}{1+(\underline{r}-\rho) d t} \\
& -\frac{(\lambda+\mu) p^{i}\left(\frac{a^{i}}{1+(\underline{r}-\rho) d t}, \underline{r}\right)}{1+(\underline{r}-\rho) d t} \\
\frac{p_{d t}^{i}\left(a^{i}, \bar{r}\right)-p^{i}\left(a^{i}, \bar{r}\right)}{d t}= & \frac{p^{i}\left(a^{i}-\frac{(\bar{r}-\rho) a^{i} d t}{1+(\bar{r}-\rho) d t}, \bar{r}\right)-p^{i}\left(a^{i}, \bar{r}\right)}{(1+(\bar{r}-\rho) d t) d t}-\frac{(\bar{r}-\rho) p^{i}\left(a^{i}, \bar{r}\right)}{1+(\bar{r}-\rho) d t} \\
& +\frac{(\lambda+\mu) p^{i}\left(\frac{a^{i}}{1+(\underline{r}-\rho) d t}, \underline{r}\right)}{1+(\underline{r}-\rho) d t}
\end{aligned}
$$




\subsection{The Aggregate Wealth Distribution}

In the absence of macro-level uncertainty in the interest rate, i.e., $\mu=0$, the law of motion for the individual's probability function in Equations (9)-(10) also represents the law of motion for the population shares. In particular, the risks at the individual level completely cancel, so that the aggregate wealth distribution evolves according to a deterministic process. However, in the presence of macro-level uncertainty, i.e., $\mu>0$, the laws of motion for the individual's probability function in Equations (9)-(10) do not represent the laws of motion for the population shares. In particular, the risks at the individual level only cancel conditional on the macro-level shock. The aggregate wealth distribution evolves according to a stochastic process.

In order to obtain the law of motion for the aggregate wealth distribution in the presence of macro-level uncertainty, I consider the joint probability function for personal wealth and the interest rate conditional on the macro-level interest-rate shock $q_{\mu}$, denoted by $p_{d t}^{i}\left(a^{i}, r^{i} \mid q_{\mu}\right)$. The probability function is consistent with the individual's laws of motion if it satisfies

$$
\begin{aligned}
& \sum_{r^{i}} \int p_{d t}^{i}\left(a^{i}, r^{i} \mid q_{\mu}\right) \mathbb{1}_{B}\left(a^{i}, r^{i}\right) d a^{i} \\
& =\sum_{r^{i}} \int p^{i}\left(a^{i}, r^{i} \mid q_{\mu}\right) \mathbb{E}_{q_{\mu}} \mathbb{1}_{B}\left(s_{d t}^{i}\left(a^{i}, r^{i}\right)\right) d a^{i}
\end{aligned}
$$

for all open set indicator functions, where $\mathbb{E}_{q_{\mu}}$ denotes expectations conditional on the shock $q_{\mu}$. Conditional on the economy not being exposed to a macro-level interest-rate shock, i.e., $d q_{\mu}=0$, the interest rate is expected to jump to the high value $\bar{r}$ and to stay at the initial level $r^{i}$ with a probability of $\lambda d t$ and $1-\lambda d t$, respectively. Conditional on the economy being exposed to a macro-level shock, i.e., $d q_{\mu}=1$, the interest rate is expected to jump to the high value $\bar{r}$ with certainty. Therefore,

$$
\begin{aligned}
\mathbb{E}_{q_{\mu}} \mathbb{1}_{B}\left(s_{d t}^{i}\left(a^{i}, r^{i}\right)\right)= & \left(1-d q_{\mu}\right)\left\{\lambda d t \mathbb{1}_{B}\left(a^{i}+d a^{i}, \bar{r}\right)+(1-\lambda d t) \mathbb{1}_{B}\left(a^{i}+d a^{i}, r^{i}\right)\right\} \\
& +d q_{\mu} \mathbb{1}_{B}\left(a^{i}+d a^{i}, \bar{r}\right)
\end{aligned} .
$$

After applying a change of variables formula in order to integrate over $a^{i}+d a^{i}$ instead of $a^{i}$, Equation (11) simplifies to

$$
\sum_{r^{i}} \int \mathbb{1}_{B}\left(a^{i}, r^{i}\right)\left\{p_{d t}^{i}\left(a^{i}, r^{i} \mid q_{\mu}\right)-F\left(a^{i}, r^{i}, d q_{\mu}, d t\right)\right\} d a^{i}=0,
$$

where $F\left(a^{i}, \underline{r}, d q_{\mu}, d t\right)$

$$
F\left(a^{i}, \underline{r}, d q_{\mu}, d t\right)
$$

$$
=\left(1-d q_{\mu}\right)(1-\lambda d t)(1+(\underline{r}-\rho) d t)^{-1} p^{i}\left(a^{i} /(1+(\underline{r}-\rho) d t), \underline{r} \mid q_{\mu}\right)
$$

$$
F\left(a^{i}, \bar{r}, d q_{\mu}, d t\right)
$$

$$
\begin{aligned}
= & \left(\left(1-d q_{\mu}\right) \lambda d t+d q_{\mu}\right)(1+(\underline{r}-\rho) d t)^{-1} p^{i}\left(a^{i} /(1+(\underline{r}-\rho) d t), \underline{r} \mid q_{\mu}\right) . \text { This condi- } \\
& +(1+(\bar{r}-\rho) d t)^{-1} p^{i}\left(a^{i} /(1+(\bar{r}-\rho) d t), \bar{r} \mid q_{\mu}\right)
\end{aligned}
$$

tion is obviously satisfied for all open set indicator functions if the terms in brackets vanish. As the time period $d t$ approaches zero, one obtains the law of 
motion for the probability function $p^{i}\left(a^{i}, r^{i} \mid q_{\mu}\right)$.

Being conditionally independent given the macro-level shock, the micro-level risks of the individuals completely cancel at the aggregate level provided that the economy is sufficiently large. Hence, the law of motion for the probability function $p^{i}\left(a^{i}, r^{i} \mid q_{\mu}\right)$ coincides with that for the aggregate distribution $p(a, r)$ :

$$
\begin{aligned}
d p(a, \underline{r})= & -(\underline{r}-\rho) a \frac{\partial p}{\partial a}(a, \underline{r}) d t-(\underline{r}-\rho) p(a, \underline{r}) d t \\
& -\lambda p(a, \underline{r}) d t-p(a, \underline{r}) d q_{\mu} \\
d p(a, \bar{r})= & -(\bar{r}-\rho) a \frac{\partial p}{\partial a}(a, \bar{r}) d t-(\bar{r}-\rho) p(a, \bar{r}) d t \\
& +\lambda p(a, \underline{r}) d t+p(a, \underline{r}) d q_{\mu}
\end{aligned}
$$

In many respects, the law of motion for the aggregate distribution $p(a, r)$ in Equations (13)-(14) is similar to the law of motion for the individual's probability function $p^{i}\left(a^{i}, r^{i}\right)$ in Equations (9)-(10). The first two terms on the right-hand side of the equations, which reflect the wealth accumulation in a given interest-rate regime, are common to both laws of motion. Furthermore, the changes in the distributions in response to the micro-level interest-rate shocks $q_{\lambda}^{i}$ have the same structure. Indeed, in the absence of the macro-level shock $q_{\mu}$, the law of motion for the aggregate distribution $p(a, r)$ coincides with that for the individual's probability function $p^{i}\left(a^{i}, r^{i}\right)$.

However, the laws of motion for the aggregate distribution $p(a, r)$ and for the individual's probability function $p^{i}\left(a^{i}, r^{i}\right)$ differ in some notable respects. At the individual level, the micro-level shock $q_{\lambda}^{i}$ and the macro-level shock $q_{\mu}$ affect the probability distribution in a similar way. Both interest-rate shocks continuously induce gradual changes in the probability function over time. At the aggregate level, the micro-level shock $q_{\lambda}^{i}$ continuously induces gradual changes in the aggregate distribution over time as well. However, the macro-level shock $q_{\mu}$ causes discrete changes in the aggregate distribution once it materializes. In particular, all individuals in the low interest-rate state $\underline{r}$ instantaneously transit to the high interest-rate state $\bar{r}$.

Most notably, the law of motion for the aggregate distribution $p(a, r)$ is a stochastic process in contrast to the deterministic law of motion for the individual's probability function $p^{i}\left(a^{i}, r^{i}\right)$. Figure 1 illustrates the evolution over time of the wealth distribution, $p(a)=p(a, \underline{r})+p(a, \bar{r})$, for two different scenarios. In the first scenario on the left-hand side of Figure 1, the economy is not exposed to a macro-level interest-rate shock over the entire time span. The individuals, who are all in the low interest-rate state $\underline{r}$ initially, dissave until eventually transiting at rate $\lambda$ to the high interest-rate state $\bar{r}$ in response to the micro-level shocks. After entering the high interest-rate state, the individuals start to accumulate wealth. The wealth distribution stretches out over time as some individuals reduce their wealth and some individuals increase their wealth. In the second scenario on the right-hand side of Figure 1, the economy is exposed to a 

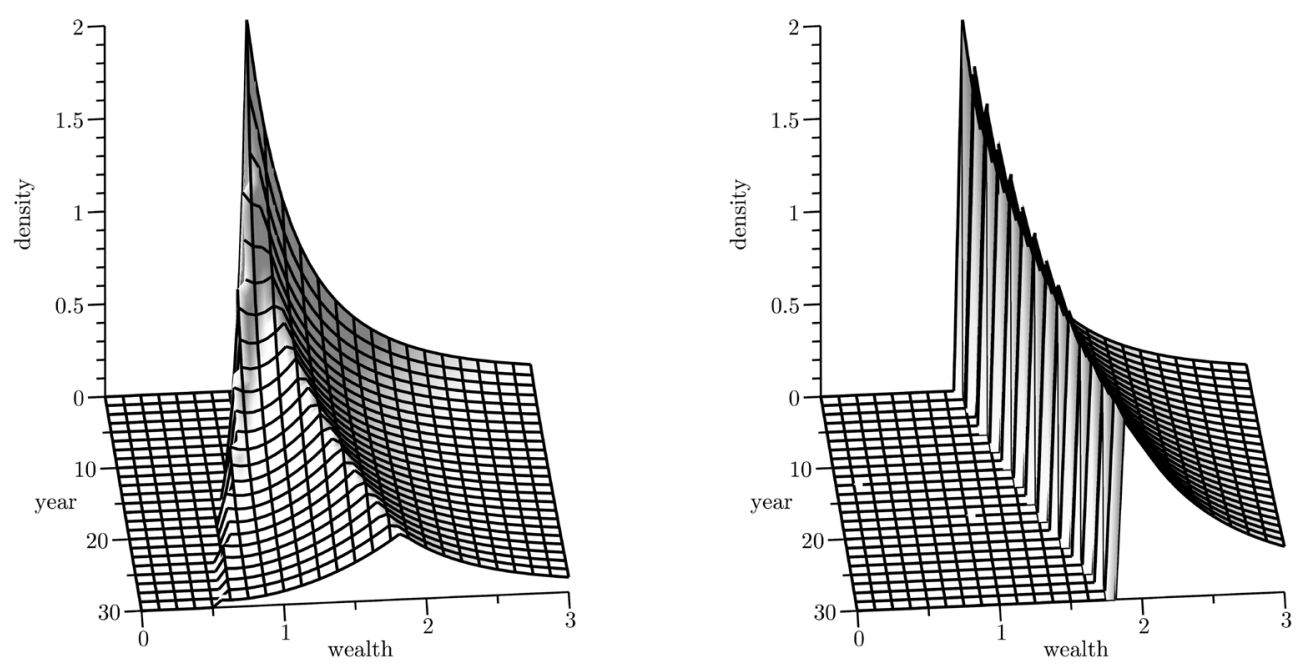

Figure 1. Evolution over time of the wealth distribution, $p(a)=p(a, \underline{r})+p(a, \bar{r})$, without (on the left-hand side) and with (on the right-hand side) a macro-level interest-rate shock, $q_{\mu}$, in the initial period. Initial distribution: $p(a, \underline{r})=2 a^{-3}$ for $a \geq 1$ and $p(a, \bar{r})=0$. Parameters: $\rho=0.05$, $\underline{r}=0.03, \bar{r}=0.07$ and $\lambda=0.15$.

macro-level interest-rate shock in the initial period, so that all individuals are in the high interest-rate state $\bar{r}$ from that point on. Therefore, all individuals save and accumulate wealth over time. The aggregate wealth distribution shifts to higher wealth levels over time.

\section{Conclusion}

In the present paper, I derive the law of motion for the aggregate distribution directly from the individuals' laws of motion. The formalism offers sufficient guidance also in more elaborate frameworks. Nevertheless, by relying on concepts from measure theory, the derivation remains both concise and intuitive. I illustrate the formalism in a model of wealth accumulation with stochastic interest rates, deriving the law of motion for the aggregate wealth distribution. ${ }^{4}$

\section{Acknowledgements}

I thank Denis Stijepic, Klaus Wälde, an anonymous referee and the participants of the Chair in Macroeconomics Research Seminar at the Johannes Gutenberg University (Mainz, 2017) for helpful comments. I gratefully acknowledge the financial support provided by the Fritz Thyssen Foundation under the grant No. 40.16.0.028WW and by the German Research Foundation (DFG) under the grant No. 433336278. The usual disclaimer applies.

\section{Conflicts of Interest}

The authors declare no conflicts of interest regarding the publication of this paper.

${ }^{4}$ While I assume that the agents in the economy know the properties of the stochastic processes, alternative setups provide further insights (see, e.g., Liu, 2010; Chen et al., 2017a, 2017b). 


\section{References}

Achdou, Y., Han, J., Lasry, J. M., Lions, P. L., \& Moll, B. (forthcoming). Income and Wealth Distribution in Macroeconomics: A Continuous-Time Approach. Review of Economic Studies.

Bayer, C., \& Wälde, K. (2010). Matching and Saving in Continuous Time: Theory. CESifo Working Paper Series 3026, Munich: CESifo Group.

Benhabib, J., \& Bisin, A. (2018). Skewed Wealth Distributions: Theory and Empirics. Journal of Economic Literature, 56, 1261-1291. https://doi.org/10.1257/jel.20161390

Cao, D., \& Luo, W. (2017). Persistent Heterogeneous Returns and Top End Wealth Inequality. Review of Economic Dynamics, 26, 301-326. https://doi.org/10.1016/j.red.2017.10.001

Chen, L., Peng, J., Liu, Z., \& Zhao, R. (2017a). Pricing and Effort Decisions for a Supply Chain with Uncertain Information. International Journal of Production Research, 55, 264-284. https://doi.org/10.1080/00207543.2016.1204475

Chen, L., Peng, J., \& Zhang, B. (2017b). Uncertain Goal Programming Models for Bicriteria Solid Transportation Problem. Applied Soft Computing, 51, 49-59. https://doi.org/10.1016/j.asoc.2016.11.027

Duffie, D., \& Sun, Y. (2012). The Exact Law of Large Numbers for Independent Random Matching. Journal of Economic Theory, 147, 1105-1139. https://doi.org/10.1016/j.jet.2012.01.003

Gabaix, X., Lasry, J. M., Lions, P. L., \& Moll, B. (2016). The Dynamics of Inequality. Econometrica, 84, 2071-2111. https://doi.org/10.3982/ECTA13569

He, W., Sun, X., \& Sun, Y. (2017). Modeling Infinitely Many Agents. Theoretical Economics, 12, 771-815. https://doi.org/10.3982/TE1647

Jacod, J., \& Protter, P. (2004). Probability Essentials. 2nd Edition, Berlin: Springer. https://doi.org/10.1007/978-3-642-55682-1

Liu, B. (2010). Uncertainty Theory: A Branch of Mathematics for Modeling Human Uncertainty. Berlin: Springer.

Merton, R. C. (1975). An Asymptotic Theory of Growth under Uncertainty. Review of Economic Studies, 42, 375-393. https://doi.org/10.2307/2296851

Moscarini, G. (2005). Job Matching and the Wage Distribution. Econometrica, 73, 481-516. https://doi.org/10.1111/j.1468-0262.2005.00586.x

Qiao, L., Sun, Y., \& Zhang, Z. (2016). Conditional Exact Law of Large Numbers and Asymmetric Information Economies with Aggregate Uncertainty. Economic Theory, 62, 43-64. https://doi.org/10.1007/s00199-014-0855-6

Sun, Y. (2006). The Exact Law of Large Numbers via Fubini Extension and Characterization of Insurable Risks. Journal of Economic Theory, 126, 31-69.

https://doi.org/10.1016/j.jet.2004.10.005 


\section{Appendix A. Proofs}

Proof of Corollary 1. The first direction is trivial. The proof of the second direction is as follows. If the condition holds for the open set indicator functions, the two measures are identical on all those open sets. By the Monotone Class Theorem, it follows that the two measures are also identical on all Borel sets, since the Borel $\sigma$-algebra is induced by those open sets and the set of those open sets is itself closed under finite intersections (see, e.g., Jacod and Protter, 2004).

\section{Appendix B. An Illustrative Example}

The interest rate that the person $i$ faces, denoted by $r^{i}$, is either low, $r^{i}=\underline{r}$, or high, $r^{i}=\bar{r}$, where $\underline{r}, \bar{r} \in \mathbb{R}^{+}$and $\bar{r}>\underline{r}$. Let $P^{i}(\cdot)$ denote the probability function that assigns probabilities to all the subsets of the state space $B \subset \Omega=\{\underline{r}, \bar{r}\}$. Furthermore, $p^{i}(\cdot)$ is the associated elementary probability function that assigns probabilities to the elements in the state space $r^{i} \in \Omega$, i.e., $p^{i}\left(r^{i}\right)=P^{i}\left(\left\{r^{i}\right\}\right)$. Let $t \in \mathbb{R}^{+}$denote time and let $d t>0$ be an infinitesimal time period.

The evolution over time of a person's interest rate is described by the Markovian stochastic process $r_{d t}^{i}\left(r^{i}\right)$. In particular, a person's current interest rate $r^{i}$ determines the probability that the person's interest rate $r_{d t}^{i}$ will be in the set $B$ at the future point in time $d t$, denoted by $P_{d t}^{i}(B)$. Specifically, I assume that a person's interest rate jumps to the high value $\bar{r}$ according to a Poisson process at rate $\lambda>0$, denoted by $q_{\lambda}^{i}$. Formally, a person's interest rate evolves according to the stochastic differential equation

$$
d r^{i}=\left(\bar{r}-r^{i}\right) d q_{\lambda}^{i},
$$

where $d r^{i}=r_{d t}^{i}-r^{i}$. I note that the high interest-rate state, $\bar{r}$, is an absorbing state. After entering that state, a person will always face the high interest rate with certainty.

\section{B.1. Probabilities and the Probability Function}

By construction, the probability that a person's interest rate will belong to the set $B$ at the future point in time $d t, P_{d t}^{i}(B)$, equals the sum of all the elementary probabilities that correspond to the elements in $B$, i.e.,

$$
P_{d t}^{i}(B)=\sum_{r^{i} \in B} p_{d t}^{i}\left(r^{i}\right)=\sum_{r^{i} \in \Omega} \mathbb{1}_{B}\left(r^{i}\right) p_{d t}^{i}\left(r^{i}\right),
$$

where $\mathbb{1}_{B}\left(r^{i}\right)$ is an indicator function that equals one if the interest rate $r^{i}$ belongs to the set $B$, i.e., $\quad r^{i} \in B$, but is zero otherwise. An alternative expression for the probability that a person's interest rate will be in the set $B$ at the future point in time $d t$ is

$$
P_{d t}^{i}(B)=\sum_{r^{i} \in \Omega} p^{i}\left(r^{i}\right) \mathbb{E} \mathbb{1}_{B}\left(r_{d t}^{i}\left(r^{i}\right)\right)
$$

where $\mathbb{E}$ denotes expectations. Intuitively, the probability conditional on the 
current interest rate $r^{i}$ that a person's interest rate $r_{d t}^{i}$ will be in the set $B$ is simply $\mathbb{E}_{B}\left(r_{d t}^{i}\left(r^{i}\right)\right)$. Furthermore, the unconditional probability that a person's interest rate $r_{d t}^{i}$ will be in the set $B$ equals the sum over all conditional probabilities weighted by the initial probability function.

An important feature of Equation (3) in contrast to Equation (2) is that expressions for the terms on the right-hand side are essentially available. Expectations of functions of a person's interest rate readily follow from the stochastic differential Equation (1). In particular, the expected future interest rate is $\mathbb{E} r_{d t}^{i}\left(r^{i}\right)=(1-\lambda d t) r^{i}+\lambda d t \bar{r}$. It follows for the function $\mathbb{1}_{B}(\cdot)$ of the interest rate that $\mathbb{E}_{B}\left(r_{d t}^{i}\left(r^{i}\right)\right)=(1-\lambda d t) \mathbb{1}_{B}\left(r^{i}\right)+\lambda d t \mathbb{1}_{B}(\bar{r})$. Equating Equation (2) and Equation (3) yields a recursive formula for the probability function $p_{d t}^{i}(\cdot)$ :

$$
\sum_{r^{i} \in \Omega} \mathbb{1}_{B}\left(r^{i}\right) p_{d t}^{i}\left(r^{i}\right)=\sum_{r^{i} \in \Omega} p^{i}\left(r^{i}\right)\left\{(1-\lambda d t) \mathbb{1}_{B}\left(r^{i}\right)+\lambda d t \mathbb{1}_{B}(\bar{r})\right\}
$$

\section{B.2. The Evolution over Time of the Probability Function}

Starting from Equation (4), I obtain the following condition after collecting terms

$$
\mathbb{1}_{B}(\underline{r})\left\{p_{d t}^{i}(\underline{r})-(1-\lambda d t) p^{i}(\underline{r})\right\}+\mathbb{1}_{B}(\bar{r})\left\{p_{d t}^{i}(\bar{r})-p^{i}(\bar{r})-\lambda d t p^{i}(\underline{r})\right\}=0,
$$

which is obviously satisfied for any arbitrary set $B$ if the terms in brackets vanish. In other words, the probability implied by the probability function $p_{d t}^{i}(\cdot)$ in Equation (2) coincides with that implied by the stochastic processes $r_{d t}^{i}(\cdot)$ in Equation (3) for any set $B$ provided that $p_{d t}^{i}(\underline{r})-(1-\lambda d t) p^{i}(\underline{r})=0$ and $p_{d t}^{i}(\bar{r})-p(\bar{r})-\lambda d t p^{i}(\underline{r})=0$.

Therefore, a probability function $p^{i}(\cdot)$ that is consistent with the stochastic differential Equation (1) evolves according to the laws of motion

$$
d p^{i}(\underline{r})=-\lambda p^{i}(\underline{r}) d t \text { and } d p^{i}(\bar{r})=\lambda p^{i}(\underline{r}) d t,
$$

where $d p^{i}(\cdot)$ denotes $p_{d t}^{i}(\cdot)-p^{i}(\cdot)$. Those laws of motion are the so-called Fokker-Planck equations or Kolmogorov forward equations for the stochastic process $r_{d t}^{i}(\cdot)$. Intuitively, the first law of motion captures the flows out of the low interest-rate state, whereas the second law of motion captures the flows into the high interest-rate state. I note that $\sum_{r^{i} \in \Omega} d p^{i}\left(r^{i}\right)=d p^{i}(\underline{r})+d p^{i}(\bar{r})=0$, i.e., the system preserves its probability mass over time.

\section{B.3. Aggregation}

An important effect of aggregation is that the micro-level risks completely cancel at the aggregate level provided that a law of large numbers applies. In particular, if the individual-level shocks $q_{\lambda}^{i}$ are independently and identically distributed and if there are sufficiently many individuals in the economy, the laws of motion for a person's probability function $p^{i}(\cdot)$ in Equation (6) coincide with those for the aggregate distribution $p(\cdot)$, i.e., 


$$
d p(\underline{r})=-\lambda p(\underline{r}) d t \text { and } d p(\bar{r})=\lambda p(\underline{r}) d t .
$$

While the former distribution represents probabilities of specific events, the latter distribution represents shares of the population in specific states. 